\title{
A adesão ao tratamento anti-hipertensivo pelo idoso: Facilitadores e dificultantes
} na visão do cuidador

\section{Adherence to anti-hypertensive treatment by the elderly: Facilitators and difficulties in the career's view}

Francinete das Graças Albuquerque Sousa1, Keila Rodrigues de Albuquerque ${ }^{2}$, Antonio Werbert Silva da Costa $^{3}$, Amanda Pereira de Azevedo4, Maria Clara Mousinho Silva Rodrigues ${ }^{5}$, Priscilla Dantas Almeida ${ }^{6}$

\section{RESUMO}

Este estudo teve o objetivo de descrever os facilitadores e dificultadores da adesão ao tratamento anti-hipertensivo pelo idoso, na visão de cuidador. Trata-se de um estudo exploratório e qualitativo com cuidadores de idosos, na área da Estratégia Saúde da Família do bairro Todos os Santos, em Teresina - Piauí, com a participação de 17 cuidadores de idosos. A coleta de dados foi realizada no domicílio, e iniciada apenas após a aceitação do participante por meio da assinatura do Termo de Consentimento Livre e Esclarecido, e realizado através de roteiro de entrevista semiestruturada elaborado pelos pesquisadores, com o uso de equipamento tipo $\mathrm{mp} 3$ para gravação das perguntas e respostas. Os dados foram tratados por meio da análise de conteúdo, de forma temática. Após análise, foram estabelecidas categorias para discussão. Dentre os principais fatores facilitadores estão a qualidade da assistência prestada, os programas de distribuição de medicamentos do governo, a escolaridade e o acolhimento familiar. E os dificultantes estão o comprometimento da renda, a assistência prestada pelos profissionais quando descritas como de baixa qualidade, 0 vínculo direto de seu tratamento à uma Unidade de Saúde, a incapacidade do prosseguimento do medicamento por déficits cognitivos, como também o convívio familiar e apoio familiar inadequado para o uso contínuo das medicações.

Palavras-chave: Hipertensão. Cuidador. Adesão à medicação.

\section{ABSTRACT}

This study aimed to describe the facilitators and complicators of adherence to antihypertensive treatment by the elderly, from the caregiver's perspective. This is an exploratory and qualitative study with elderly caregivers, in the area of the Family Health Strategy of the Todos os Santos neighborhood, in Teresina - Piauí, with the participation of 17 elderly caregivers. Data collection was performed at home and started only after the participant's acceptance by signing the Free and Informed Consent Form, and carried out through a semi-structured interview script prepared by the researchers with the use of $\mathrm{mp} 3$ equipment to record the questions and answers. The data were treated by content analysis, in a thematic way. After the analysis, categories were established for discussion. Among the main facilitating factors are the quality of the assistance provided, the government drug distribution programs, the level of education, and the family welcoming. And the complicating factors are the compromised income, the assistance provided by professionals when described as low quality, the direct link of their treatment to a Health Unit, the inability to continue the medication due to cognitive deficits, as well as family life and inadequate family support for the continued use of medications.

Keywords: Hypertension. Caregiver. Adherence to medication. 


\section{INTRODUÇAOO}

Em um contexto de transição demográfica, a população mundial vem apresentando número cada vez maior de pessoas acima de 60 anos (SCHIMIT et al., 2011). No Brasil, a população vem envelhecendo de forma rápida desde o início da década de 60 , em acompanhamento às mudanças nas políticas de saúde e de caráter social que alteraram a distribuição etária modificando a base da pirâmide populacional nos últimos anos (MIRANDA; MENDES; SILVA, 2016).

Com o surgimento de políticas públicas, houve uma extensa modificação no cenário quanto aos tipos de doenças e agravos mais prevalentes passando a ocupar o topo da lista as enfermidades crônicas e não transmissíveis. A mudança na expectativa de vida da população, o fenômeno do envelhecimento e o aumento rápido das doenças crônicas não-transmissíveis (DCNT) típicas dos idosos, fizeram com que a saúde pública sofresse uma reestruturação em suas ações (OLIVEIRA, 2013; SANTOS et al., 2010).

Para tanto, o governo realizou inúmeros programas na área, desenvolvendo ações de promoção, proteção, prevenção e manutenção da saúde para idosos hipertensos, diabéticos e portadores de outras DCNT por meio da Estratégia Saúde da Família (ESF), buscando a manutenção da saúde dessa população (BRASIL, 2013).

Das várias comorbidades típicas dessa faixa etária, uma das mais preocupantes é a Hipertensão Arterial Sistêmica (HAS) doença crônica em que a pressão sanguínea nas artérias encontra-se constantemente elevada e por ser uma doença silenciosa que não causa sintomas em seu início, em longo prazo torna-se um dos principais fatores de risco para uma série de doenças cardiovasculares graves (SBC, 2016).

As principais causas da má adesão ao tratamento são fatores relacionados a aspectos inerentes ao paciente, questões que envolvem o próprio sistema de saúde e fatores associados à doença e ao tratamento, e no que diz respeito ao paciente idoso com HAS, associam-se a baixa adesão ao tratamento o esquecimento, o esquema terapêutico com o uso de várias medicações, barreiras financeiras e outros aspectos socioeconômicos (OLIVEIRA, 2013; GUS et al., 2013).

Assim esse trabalho tem a finalidade de analisar de forma qualitativa quais são os facilitadores e dificultantes da adesão ao tratamento anti-hipertensivo pelo idoso, na visão do cuidador. 


\section{MATERIAIS E METODOS}

Trata-se de um estudo exploratório de abordagem qualitativa desenvolvida na região sudeste da cidade de Teresina, em domicílios da área de abrangência da ESF lotada em Unidade Básica de Saúde (UBS) localizada no bairro Todos os Santos.

Participaram do estudo 17 cuidadores de idosos, que faziam parte da população adscrita da ESF. Foram critérios de inclusão do estudo ser cuidador de idosos com diagnóstico de HAS há pelo menos 6 meses e que exerciam atividade do cuidar por no mínimo 12 horas diárias. Foram considerados critério de exclusão aqueles cuidadores que exerciam a função de forma esporádica ou que estivessem a menos de seis meses exercendo a atividade do cuidar.

A coleta de dados foi realizada no domicílio, e iniciada apenas após a aceitação do participante por meio da assinatura do Termo de Consentimento Livre e Esclarecido, e realizado através de roteiro de entrevista semiestruturada elaborado pelos pesquisadores, com o uso de equipamento tipo mp3 para gravação das perguntas e respostas. Os dados foram tratados por meio da análise de conteúdo, de forma temática, sendo estabelecidas categorias homogêneas, ou seja, todas foram obtidas a partir dos mesmos princípios (MINAYO et al., 2015).

A pesquisa foi realizada em conformidade com a Resolução do Conselho Nacional de saúde no 466/12, que trata sobre pesquisas envolvendo seres humanos, obtendo-se aprovação da instituição, como também aprovado pelo Comitê de Ética e Pesquisa da Universidade Paulista, conforme CAAE № 94660718.0.0000.5512.

\section{RESULTADOS E DISCUSSĀO}

As características socioeconômicas que mais predominaram nos participantes foram indivíduos do sexo feminino (15), na faixa etária de 31 a 42 anos (6) e acima de 55 anos de idade (7), com ensino médio completo (7) e renda familiar entre 1 a 2 salários mínimos mensais (12), exercendo a função de cuidador há mais de 4 anos e sendo estes, em sua maioria filhos, netos, noras, ou ainda não apresentando nenhum parentesco com o idoso, conforme Tabela 01.

A quantidade de pessoas de sexo feminino está diretamente relacionada ao fato de as entrevistadas serem mulheres do lar sem trabalho fixo, o que faz com que passem a maior parte do tempo em casa cuidando dos afazeres domésticos assim como das crianças e idosos. Esse fato já vem sendo evidenciado em alguns trabalhos tais como o de 
Scarpellini et al. (2012) e Oliveira (2013) que tratam do perfil dos cuidados de idosos hipertensos.

Os dois únicos participantes do sexo masculino presentes na pesquisa enquadravamse como filhos dos idosos e estavam em situação de desemprego no momento da entrevista, motivo este que justificou sua estadia na casa dos pais na ocasião da coleta de dados.

Tabela 01: Características socioeconômicas dos cuidadores de idosos entrevistados na região sudeste de Teresina - PI, 2018.

\begin{tabular}{|c|c|c|}
\hline CARACTERÍSTICA & CATEGORIA & QUANTIDADE \\
\hline \multirow[t]{4}{*}{ Faixa Etária } & $18 a-30 a$ & 01 \\
\hline & $31 a-42 a$ & 06 \\
\hline & $43 a-54 a$ & 03 \\
\hline & Acima de $55 a$ & 07 \\
\hline \multirow[t]{2}{*}{ Sexo } & Feminino & 15 \\
\hline & Masculino & 02 \\
\hline \multirow[t]{5}{*}{ Estado Civil } & Casado (a) & 11 \\
\hline & Solteiro (a) & 04 \\
\hline & Divorciado (a) & 00 \\
\hline & Viúvo (a) & 00 \\
\hline & União Estável & 02 \\
\hline \multirow[t]{7}{*}{ Escolaridade } & Analfabeto & 02 \\
\hline & Ens. Fund. Incompleto & 05 \\
\hline & Ens. Fund. Completo & 02 \\
\hline & Ens. Méd. Incompleto & 00 \\
\hline & Ens. Méd. Completo & 07 \\
\hline & Super. Incompleto & 01 \\
\hline & Super. Completo & 00 \\
\hline \multirow[t]{5}{*}{ Tempo na função de cuidador } & $6 m-1 a$ & 02 \\
\hline & $1 a-2 a$ & 01 \\
\hline & $2 a-3 a$ & 03 \\
\hline & $3 a-4 a$ & 03 \\
\hline & Acima de $4 a$ & 08 \\
\hline \multirow[t]{4}{*}{ Renda Familiar } & < 1 salário mínimo & 02 \\
\hline & 1 a 2 salários mínimos & 12 \\
\hline & 2 a 3 salários mínimos & 01 \\
\hline & >3salários mínimos & 02 \\
\hline \multirow[t]{5}{*}{ Grau de Parentesco com o idoso } & Pai/mãe & 00 \\
\hline & Irmão/irmã & 00 \\
\hline & Filho & 07 \\
\hline & Tio/tia & 00 \\
\hline & $\begin{array}{c}\text { Outros (neto, nora, genro, enteado, } \\
\text { nenhum) }\end{array}$ & 10 \\
\hline
\end{tabular}

Fonte: Pesquisa própria.

Após análise dos dados coletados foi possível estabelecer categorias a fim de melhor esclarecer os objetivos propostos, sendo, portanto, assim definidas: 5 (cinco) facilitadores: Medicamentos ofertados pelo SUS e entregues regularmente na UBS aos idosos 
hipertensos; Rotina de consulta domiciliar exercidas; Presença constante do Agente comunitário de saúde na comunidade; Autonomia positiva do idoso e colaboração favorável em relação a aceitação do tratamento; Cuidado, afeto, atenção e boa vontade dos familiares mais próximos; e 6 (seis) dificultadores: Comportamento do idoso em relação ao tratamento; Dificuldade de deslocamento tanto individualmente como com o idoso; Dificuldade financeira para suprir medicação extra tanto para HAS como para outras doenças crônicas que o idoso apresente; Presença de outras doenças que dificultam o cuidado e progressão do tratamento; Demora na entrega das medicações pela UBS; e Falta de apoio familiar.

Para respeitar os aspectos éticos e legais e garantir o anonimato dos participantes os nomes verdadeiros foram omitidos e substituídos pela letra " $E$ " de "entrevistado" destacando-se as numerações conforme a ordem na qual as entrevistas foram ocorrendo, começando com "E1" (Entrevistado No1) e finalizando com "E17" (Entrevistado №17).

\subsection{FACILITADORES DA ADESÃO AO TRATAMENTO ANTI-HIPERTENSIVO NA PESSOA IDOSA}

Quando o indivíduo doente vai ao posto médico em busca de tratamento ele objetiva pôr fim a um mal que o está afligindo, além disso, ele espera ser tratado de forma respeitosa, adequada e com total responsabilidade que the cabe em direito. Assim, a qualidade da assistência prestada nos estabelecimentos de saúde é de suma importância para a melhoria das condições clínicas do indivíduo e em casos de pacientes crônicos, ela se torna um ponto chave para que a o tratamento da doença progrida de forma satisfatória (BRASIL, 2013).

É inegável que a gama de programas desenvolvidos pelo Ministério da Saúde para o tratamento e controle das doenças crônicas relacionadas à pessoa idosa tenha favorecido a adesão ao tratamento da HAS. No entanto, além da criação desses programas é necessário que haja uma boa implementação das ações e estratégias nas redes de Atenção Básica de atendimento para que de fato ocorra uma prestação de assistência de qualidade e que esta assistência se torne eficaz para a manutenção do tratamento das doenças crônicas, sobretudo da HAS (BRASIL, 2013).

No caso da região pesquisada, na zona de abrangência da UBS são implantadas estratégias tais como realização de visitas domiciliares periódicas, entrega gratuita de 
medicamentos de uso contínuo e Hiperdia, programas estes, que auxiliam bastante no acolhimento de idosos portadores de hipertensão e favorecendo a melhor progressão do tratamento, uma vez que, por meio do acompanhamento diário desses casos se torna ainda mais fácil verificar a rotina do indivíduo e avaliar se este está de fato seguindo as orientações necessárias para a continuidade de tratamento de forma correta e satisfatória (BARBOSA et al., 2012). Essa situação é bem explicitada nos comentários abaixo:

"[...] As medicações, quando têm, ajudam muito por que ai nós não gastamos com os comprimidos dela (idosa). [...] a agente de saúde que está sempre vindo aqui onde a gente, sempre ajuda nas consultas, também é bom. Os médicos vêm aqui mais demoram um pouco, mais pelo menos vêm também[...]" (Participante E1).

Além disso, tais programas e estratégias são de grande valia para a população, por evitar que os pacientes crônicos tenham gastos desnecessários com a compra do medicamento para tratamento e com deslocamentos periódicos ao posto da saúde. Isso, sem dúvida, é uma vantagem para a vida financeira não só do idoso, mas de toda sua família.

"[...] O remédio que eles entregam no postinho facilita muito, por que nós não precisamos comprar os comprimidos, antes era ruim ter que comprar toda vez [...]" (Participante E3).

Por se tratar de pacientes idosos o deslocamento, muitas vezes, é difícil e, dependendo da mobilidade do indivíduo pode se tornar um incômodo não só para o próprio idoso hipertenso como para quem o está transportando. Isso é ruim e interfere na continuidade do tratamento, ao passo que, o indivíduo, por causa de tal situação, deixa de comparecer às consultas e/ou de receber as medicações para tratamento da doença por causa da falta de dinheiro ou tempo para se deslocar de sua residência até a UBS, principalmente se o hipertenso morar longe do posto. Por isso, as visitas domiciliares e a presença do agente comunitário de saúde influenciam na adesão ao tratamento antihipertensivo.

O envelhecimento é uma fase da vida na qual o indivíduo passa por alterações físicas, psicológicas e emocionais (LIMA, 2016). Neste estudo foram confirmados casos em que o idoso ainda mantinha sua plena autonomia e independência de autocuidado e 
seguia o tratamento de forma regular, fazendo uso dos remédios de forma adequada e dentro dos horários, mesmo quando se tratava de muitas medicações (Queiroz et al., 2014).

"[...] Ele sabe. Os remédios, ele (idoso) toma todos sozinho, já fica tudo em cima da mesinha. Eu só fico de olho para ver se ele toma direito, mas ele toma tudo [...]". (Participante E1).

"[...] Ela (idosa) já sabe, os remédios dela já ficam aqui todos separados nas cartelinhas, ai ela toma o diurético junto com o enalapril [...]". (Participante E17).

Esses idosos demonstravam um comportamento favorável, contribuindo de forma positiva para a tomada da medicação, o que reduzia os sinais e sintomas da HAS bem como favorecia a melhoria da própria qualidade de vida afastando e/ou minimizando assim as possíveis complicações da doença.

Essas pessoas idosas, assim como seus cuidadores tinham grau de escolaridade elevado (nível médio ou superior), demonstrando ter conhecimento sobre a doença e além disso, a renda familiar média era acima de 2 (dois) salários mínimos e o idoso realizava regularmente algum tipo de atividade física e mantinha uma alimentação saudável como forma de auxiliar no controle dos níveis pressóricos.

Por fim, o último ponto citado como fator favorável a uma boa adesão pelos entrevistados foi o apoio familiar e os sentimentos desencadeados pela relação de cuidado desenvolvida durante a realização do tratamento (BARRETO; MARCON, 2014). De acordo com Costa e Nogueira (2011), O apoio da família representa para a maioria das pessoas uma importante fonte de apoio e segurança, permitindo troca de amor, afeto, respeito e valores. Portanto, a familiar e suas interações influenciam diretamente no sucesso do tratamento da HAS.

Assim, é importante que o cuidador e todos da família desenvolvam sentimentos de amor e empatia em relação ao idoso hipertenso e que sintam a necessidade de cuidar dele não simplesmente como uma tarefa que deva ser executada de forma automática, mas como alguém que, por fazer parte do seio familiar, lhe seja dado a devida importância pelas contribuições já prestadas àquele lar, sendo tratado com o carinho, amor e respeito que merece pelos demais membros da família. É o que sugere o comentário abaixo:

"[...] Primeiro de tudo, eu acho, que o cuidador, seja filho, seja filha, seja uma pessoa que é paga para cuidar, ele tem que ter amor no coração e boa vontade. Por que se você não tiver boa vontade, você faz tudo no supetão. Graças a Deus aqui não acontece isso. Minha mãe não me dá trabalho e toma todos os remédios, direitinho [...]". (Participante E2). 
O apoio familiar torna-se um suporte à adesão, ao passo que, minimiza o risco para o desenvolvimento de outras patologias (LOPES; MARCON, 2013). Na opinião dos cuidadores, a relação familiar, sem dúvida, é a chave para uma boa aceitação da doença bem como para a progressão satisfatória do tratamento e controle dos níveis pressóricos:

"[...] Eu acho que se a familia fosse mais próxima a ele (idoso) acho ia facilitar a doença dele, ele iria aceita melhor a doença e iria querer ficar bom, querer tomar os remédios [...]". (Participante E1).

Desse modo, o contexto familiar estruturado é apontado como significativamente associado ao comportamento de autocuidado na doença crônica, por exercer importante papel em todo o processo de relação do indivíduo com a sua doença, seu tratamento e a adaptação a um novo estilo de vida (BARRETO; MARCON, 2014).

\subsection{DIFICULTADORES DA ADESÃO AO TRATAMENTO ANTI-HIPERTENSIVO PELA PESSOA IDOSA}

Como já citado anteriormente, a qualidade da assistência prestada ao idoso hipertenso na UBS, assim como os programas desenvolvidos pelos órgãos de saúde são importantes para uma progressão satisfatória do tratamento anti-hipertensivo. Entretanto, apesar de tais implementações, ainda ocorrem algumas falhas no atendimento prestado. Esses problemas, na maioria das vezes, estão relacionados a fatores administrativos e de gestão e envolvem, sobretudo, questões financeiras (OLIVEIRA, 2013).

Nesse contexto, o estudo demonstrou uma grande insatisfação dos cuidadores com a demora das visitas domiciliares realizadas e com a demora na chegada da medicação que é distribuída periodicamente à comunidade para o tratamento. Dos participantes entrevistados, alguns queixaram-se do fato de ter que utilizar a renda familiar para suprir as medicações e manter o tratamento dos idosos em dia. Esses dados são bastante relevantes, uma vez que se tornam um fator dificultador da adesão ao tratamento da HAS:

"[...] o problema é que a médica costuma demorar a vir. [...] Que as vezes o remédio não tem lá (no posto), aí os remédios acabam[...] às vezes é o filho dela quem compra os remédios que às vezes custa demais vir lá do posto. As vez a gente compra mais do que recebe. A gente compra muito remédio." (Participante E4). 
Para sanar esse problema, é necessário que haja um esforço por parte governo, que deve desenvolver estratégias e sistemas mais seguros de fiscalização para implantação dos programas e principalmente demandando suporte financeiro e técnico para as redes de atenção básica adequarem-se às ações de promoção, proteção e recuperação da saúde para pacientes crônicos, como a distribuição regular e gratuita de medicamentos de uso contínuo e acompanhamento domiciliar periódico (BRASIL, 2013).

Por ser um processo de diminuição orgânica e funcional, não decorrente de doença, o envelhecimento acontece inevitavelmente com o passar do tempo e é considerado como um fenômeno natural (LIMA, 2016). Para Valer et al. (2015) as mudanças no comportamento, alterações de humor, esquecimento, são situações recorrentes e relacionadas ao envelhecimento, que prejudicam bastante a continuidade do tratamento, principalmente em caso de doença crônica, onde o paciente é submetido ao uso medicações associadas por um tempo prolongado.

"[...] No começo, tinha vezes que ele (o idoso) não queria tomar o medicamento, dava trabalho, dizia que o remédio tava fazendo mal, só para não tomar, dava muito trabalho mesmo [...]". (Participante E7).

"[...] Ela não toma corretamente o remédio, às vezes, 8h, quando eu vou lá, ela ainda não tem tomado [...] e sempre foi assim, ela nunca dá valor a esse negócio de medicação. Ela sempre tem raiva de toma a medicação [...]" (Participante E9).

"[...] O medicamento que ela (idosa) toma é pela manhã e meio-dia, mas ela só está tomando de manhã, porque essa mulher aí dá trabalho, só quer as coisas do jeito dela, quando não é do jeito dela ela se zanga [...]". (Participante E13).

Outra situação que se repetiu bastante nas entrevistas foram os relatos de esquecimento quanto ao uso das medicações. Nesses casos, o cuidador tomava para si a responsabilidade de dar continuidade ao tratamento, realizando a tarefa de dar os remédios conforme prescrição e horário.

"[...] De primeiro, ela (idosa) tomava direitinho (o remédio), mas agora eu tenho que dar, porque ela fica esquecendo, ai eu tenho que dá [...]" (Participante E5).

Para Queiroz et al. (2014) o envelhecimento é uma experiência heterogênea, ou seja, pode ocorrer de forma diferente em cada indivíduo e vai depender basicamente do contexto social no qual o idoso está inserido e das influências sofridas pelas circunstâncias histórico 
culturais, dos fatores intelectuais, da personalidade, dos hábitos e atividades físicas exercidas ou não e da incidência de patologias apresentadas ao longo da vida. Assim sendo, o esquecimento está intimamente relacionado às transformações sofridas pelo indivíduo e deve ser tratado de forma natural e com compreensão por todos que convivem com o idoso para que, de forma, não se torne um influenciador da má adesão ao tratamento da HAS (OLIVEIRA, 2013).

Nesse contexto, o cuidador torna-se um eixo fundamental na adesão ao tratamento anti-hipertensivo por ser o indivíduo que está mais próximo ao idoso acompanhando de perto a execução do tratamento. Ele é um indivíduo que, na maioria das vezes compartilha do mesmo espaço sociocultural do idoso. Como mencionado por Bohm et al. (2011) a escolha para essa função vai depender de vários fatores, sendo fatores geracionais, de gênero, grau de parentesco, ter condições financeiras favoráveis, dispor de tempo, etc.

Em sua maioria, essas pessoas são parentes que convivem diariamente com a pessoa idosa e conhecem de suas limitações e doenças. Por isso mesmo, ao realizar tal tarefa cria-se uma relação de obrigação de cuidado entre o cuidador e o idoso. E dessa forma, alguns problemas acabam sendo gerados na vida e perspectiva do cuidador que, muitas vezes, sofre com as atribulações do cotidiano ao assumir tal função (BOHM et al., 2011).

A coleta e análise dos dados apontaram que apenas um dos participantes tinha curso de formação na área de cuidador de idosos, sendo, na ocasião, contratado pela família do idoso para a execução da atividade. Os demais entrevistados todos tinham apenas a relação familiar de parentesco com o idoso, mas, nenhum contava com formação na área de cuidador e nem havia recebido quaisquer orientações específicas para o cuidado com o mesmo, contando apenas com o saber - comum cotidiano. Esse aspecto é desfavorável, pois à medida que o indivíduo não tem um conhecimento apropriado do que se propõe a realizar pode gerar desqualificação no cuidado e pode provocar estagnação ou ainda involução do quadro clínico, podendo acarretar futuramente uma sobrecarga para o próprio cuidador (SCARPELLIN et al., 2012).

Outra questão evidenciada foi a dificuldade em conciliar as atividades de rotina com os cuidados prestados ao idoso. Situações como o ato de se deslocar da residência e a realização de tarefas do dia-a-dia foram destacadas nos comentários como verdadeiras barreiras enfrentadas diariamente. Como já demonstrado em relatos anteriores, o ato de se deslocar, tanto sozinho como com o idoso é uma atividade bastante exaustiva e cansativa 
e torna-se um incômodo para ambas as partes em questão, pois demanda gasto financeiro e tempo. Além disso, o cuidador, por vezes, tem que desempenhar outras atividades além do cuidado com o idoso, atividades essas que se referem a sua rotina pessoal e como membro do seio familiar:

"[...] Olha é bem ruim, porque somos só eu e ele (idoso) na casa [...] Quando eu saio, tenho que deixa sempre alguém com ele, ai é difícil por que nem sempre acho alguém desocupado na hora pra ficar. [...]"

(Participante E14).

“[...]É uma rotina diária. Tem que fazer tudo por ele (idoso), por que ele não consegue fazer nada mais [...] Agora para facilitar, assim, o acompanhamento dele, para ter tudo na horinha certinha só se eu fosse, assim, a pessoa que cuidasse somente dele, mas eu não cuido só dele. Cuido dele, cuido da casa. Têm outras atividades para fazer também [...]". (Participante E10).

Essas são situações relevantes devido ao fato de acarretar estresse para o cuidador que, por sua vez, acaba tendo que dar conta de um grande volume de atividades simultaneamente, podendo, dessa forma, esquecer ou mesmo atrasar as medicações do idoso. Nesse caso, podem ocorrer erros tais como: dar os medicamentos da maneira errada, trocando horários, repetir as mesmas dosagens várias vezes ao dia ou, ainda, confundir com outros remédios caso o idoso apresente mais de uma patologia (BARBOSA et al., 2012) o que interfere diretamente no efeito da medicação e, consequentemente, no controle dos níveis pressóricos.

Portanto, faz-se necessário a criação de esquemas terapêuticos mais práticos, rápidos e apropriados para a tomada das medicações nesses casos, a fim de não prejudicar a rotina de atividades diárias executas pelo cuidador e também não interferir na continuidade de tratamento.

O ambiente familiar é o ponto de suporte para todo e qualquer indivíduo principalmente para a pessoa idosa que, com o passar do tempo, muda com as limitações e incapacidades que o corpo vai sofrendo ao longo da vida, tornando-se cada vez mais dependente de atenção, cuidado, carinho e afeto. Daí a influência exercida pela família no decorrer do tratamento ser tão importante, sobretudo para doentes crônicos (DANTAS et al. 2012).

Contudo, se a família não der o apoio necessário, o paciente crônico tende a não aceitar bem o tratamento, o que desencadeia as alterações corporais com consequente 
involução do quadro clínico e surgimento de complicações. Segundo Costa e Nogueira (2011) a dificuldade na adesão e o desinteresse pelo tratamento aumentam quando não há envolvimento da família no cuidado diário com o doente e o relacionamento entre seus membros é conflituoso.

"[...] têm vezes que ele (idoso) diz que não vai mais (continuar o tratamento, ir às consultas). Por quê? Por que ele não tem o apoio da família. Se tivesse, ele iria tranquilo. Para que ele vai fazer um tratamento se não tem apoio? Ele se sente muito sozinho, sem a família ligar para ele [...]". (Participante E1).

No entanto, quando a família assume seu papel de cuidadora, percebe-se uma resposta rápida e satisfatória no controle da doença. Uma vez que, influenciado pela família, o idoso consegue perceber a necessidade de executar o tratamento de forma adequada para manter sua saúde em níveis de normalidade e assim está mais próximo de seus parentes, por quem nutre sentimentos de afeto (DANTAS et al., 2012), como demonstra o relato abaixo:

"[...] Ela (idosa) está bem melhor. Antes ela estava bem ruinzinha, por que a pressão dela estava todo tempo alta. Agora não. A gente cuida bem dela, dá os remédios, eu faço a comidinha dela, não deixo ela só. Fico sempre por perto. Ela tá bem melhor agora [...]". (Participante E2).

Por isso, o espaço domiciliar é visto, hoje, como um local onde pessoas com doenças crônicas, especialmente os hipertensos, podem ter boa qualidade de vida e manter a estabilidade da doença, por meio do acompanhamento, compreensão e o auxílio dos familiares na manutenção de um estilo de vida saudável (BARRETO; MARCON, 2014).

Assim, para facilitar a adesão ao tratamento e melhorar a qualidade de vida do idoso hipertenso, é necessário que haja um engajamento por parte de todos os atores envolvidos no processo saúde-doença: os idosos hipertensos, seus familiares, as pessoas de seu convívio mais próximo e os profissionais de saúde responsáveis para que o tratamento da hipertensão ocorra, de fato, de forma correta, adequada e satisfatória (DANIEL; VEIGA, 2013).

\section{CONSIDERAÇÖES FINAIS}

Este estudo permitiu identificar os principais fatores facilitadores e dificultantes para adesão medicamentosa de hipertensos na visão dos cuidadores, sendo fatores que 
facilitam a qualidade da assistência prestada, os programas de distribuição de medicamentos do governo, a escolaridade dos hipertensos e o acolhimento familiar.

Dentre os fatores que dificultaram estão o comprometimento da renda, a assistência prestada pelos profissionais quando descritas como de baixa qualidade, o vínculo direto de seu tratamento à uma UBS, a incapacidade do prosseguimento do medicamento por déficits cognitivos, como também o convívio familiar e apoio familiar inadequado para o uso contínuo das medicações.

Por se tratar de uma das doenças crônicas mais preocupantes do momento e um dos principais fatores de risco para doenças cardiovasculares, a HAS deve ser tratada com bastante atenção pelos centros de saúde, sendo necessárias ações contínuas de promoção, proteção e recuperação da saúde para a população, com vistas aos fatores que ajudem e que atrapalham na adesão medicamentosa.

É importante a transição no modelo de saúde, saindo apenas do esquema medicamentoso e biomédico e passando para um sistema que interaja e conheça os fatores relacionados a pessoa que está em tratamento, como também seus familiares e cuidadores, de forma que estes sejam integrados ativamente.

Recomenda-se novos estudos que busquem relacionar as dificuldades para adesão medicamentosa de hipertensos, pois será de grande relevância para a saúde pública a identificação e colaboração dos pesquisadores para o controle dessa condição crônica.

\section{REFERÉNCIAS}

BARBOSA, R. G. B. et al. Adesão ao tratamento e controle da pressão arterial em idosos com hipertensão. Arquivo Brasileiro de Cardiologia, Ribeirão Preto-SP, 2012.

BARRETO, M. S; MARCON, S. S. Participação familiar no tratamento da Hipertensão arterial na perspectiva do doente. Rev. Texto e Contexto de Enfermagem, vol. 23, núm. 1, Florianópolis, p. 38-46, 2014.

BOHM, V. et al. Ser cuidador de idosos: sentimentos desencadeados por esta relação. Revista Kairós Gerontologia, vol. 13, núm. 1, São Paulo-SP, p. 211-220, 2011.

BRASIL. Ministérios da Saúde. Estratégias para o cuidado da pessoa com doença crônica: hipertensão arterial sistêmica. Brasília: Ministério da Saúde, 2013.

COSTA, R. S.; NOGUEIRA, L. T. Contribuição familiar no controle da hipertensão arterial. Revista Latino Americana de Enfermagem. Vol. 16, núm. 5, p. 75-80, 2011. 
DANIEL, A. C. Q. G.; VEIGA, E. V. Fatores que interferem na adesão terapêutica medicamentosa em hipertensos. Revista Einstein, vol. 11, núm. 3, Ribeirão Preto-SP, p. 331337, 2013.

DANTAS, L. H. T. et al. Perfil da família cuidadora de idoso doente/fragilizado do contexto sociocultural de Florianópolis, SC. Revista Texto e Contexto de Enfermagem, vol. 15, núm. 4, Florianópolis-SC, p. 570-577, 2012.

GUS, I. et al. Prevalência, reconhecimento e controle da hipertensão arterial sistêmica no estado do Rio Grande do Sul. Arquivo Brasileiro de Cardiologia. Vol. 83, núm. 5,p. 424428, 2013.

LIMA, P. V. Envelhecer com dependência funcional: memória de idosos longevos. 116 f. Dissertação (Mestrado) - Universidade Estadual do Sudoeste da Bahia - Campus de Vitória da Conquista, Vitoria da Conquista, 2016.

LOPES, M. C. L.; MARCON, S. S. A vivência do idoso e sua família com a hipertensão arterial. Ciência, cuidado e saúde, vol. 12, núm. 2, p. 241-248, 2013.

MINAYO, M. C. S. et al. Pesquisa Social. 34르 ed. Petrópolis-RJ: Vozes, 2015.

MIRANDA, G. M. D.; MENDES, A. C. G.; SILVA, A. L. A. O envelhecimento populacional brasileiro: desafios e consequências sociais atuais e futuras. Revista Brasileira de Geriatria e Gerontologia, vol. 19, núm. 3, p. 507-519, 2016.

OLIVEIRA, F. P. Fatores que interferem na adesão ao tratamento anti-hipertensivo pelos idosos: revisão de literatura. $29 \mathrm{f}$. Trabalho de Conclusão de Curso (Especialização) - Universidade Federal de Minas Gerais, Araçuaí-MG, 2013.

QUEIROZ, M. M. S. et al. Envelhecimento saudável: cuidados em saúde e melhoria da qualidade de vida de idosos na atenção básica de João Pessoa-PB. Revista Ciência e Saúde Nova Esperança, vol. 12, núm. 2, 2014.

SANTOS, A. A. et al. Perfil dos cuidadores de idosos com alterações cognitivas em diferentes contextos de vulnerabilidade social. Revista Gaúcha de Enfermagem, 2010.

SOCIEDADE BRASILEIRA DE CARDIOLOGIA (SBC). VII Diretrizes brasileiras de hipertensão. Arquivo Brasileiro de Cardiologia. vol. 107, núm. 3, supl. 3, 2016.

SCARPELLINI, M. et al. A importância do cuidador de idosos na assistência ao idoso. Revista Contexto e Saúde, vol. 10, núm. 20, p. 85-92, 2012.

SCHMIDT, M. I. et al. Doenças crônicas não transmissíveis no Brasil: carga e desafios atuais. The Lancet, p. 61-73, 2011.

VALER, D. B. et al. O significado do envelhecimento saudável para pessoas idosas vinculadas a grupos educativos. Revista Brasileira de Geriatria e Gerontologia, vol. 18, núm. 4, p. 809-819, 2015. 